\title{
Incidencia de la realidad aumentada en los procesos de aprendizaje de las funciones matemáticas
}

\author{
Olga M. Martínez ${ }^{\star *}$, Ever Mejía1, William R. Ramírez² y Tarcisio D. Rodríguez ${ }^{3}$ \\ (1) Universidad de la Costa, Dpto. de Humanidades., Calle 58 No. 55-66, Barranquilla-Colombia. \\ (correo-e: omartinez@cuc.edu.co; emejia13@cuc.edu.co) \\ (2) Institución Educativa Distrital La Esperanza del Sur, Dpto. de Matemáticas, Cra. 8G \# 97-71, Barranquilla-Colombia. \\ (correo-e: wiram05@hotmail.com) \\ (3)Universidad del Atlántico, Dpto. de Matemáticas, Carrera 30 Número 8- 49 Puerto Colombia - Atlántico. \\ (correo-e: tarsiciojesusrperez@hotmail.co)
}

Recibido Sep. 25, 2020; Aceptado Nov. 24, 2020; Versión final Ene. 25, 2021, Publicado Jun. 2021

\section{Resumen}

La presente investigación tuvo como propósito determinar la incidencia de la realidad aumentada en los procesos de aprendizaje de las funciones matemáticas. Se estructuró bajo un enfoque cuantitativo de tipo explicativo, con diseño cuasi experimental y con dos grupos (experimental y control), aplicándose un test en dos momentos. Participaron 70 estudiantes de grado $9^{\circ}$ de educación básica de instituciones educativas de Barranquilla (Colombia). Se utilizó una lista de cotejo, para identificar aplicaciones informáticas de la realidad aumentada, y dos cuestionarios (pre-test y post-test) para valorar el nivel de conocimiento en el tema de funciones matemáticas. Al aplicar inicialmente el pre-test a ambos grupos se obtuvo una media homogénea. En el post-test se evidencio que los estudiantes del grupo experimental lograron superar en promedio al grupo control. Como conclusión, se determinó que la utilización de la realidad aumentada, implementada como estrategia pedagógica, permitió obtener mejores resultados en el aprendizaje de funciones de matemática en los estudiantes.

Palabras clave: realidad aumentada; aprendizaje; funciones matemáticas; estrategia pedagógica

\section{Incidence of augmented reality in the learning process of mathematical functions}

\begin{abstract}
The purpose of this research study is to determine the incidence of augmented reality in the learning process of mathematical functions. An explanatory quantitative approach and a quasi-experimental design were applied with two groups (experimental and control) that were tested in two instances. In total, 70 ninth grade students from Barranquilla (Colombia) are surveyed. Acheck list is used to identify computer applications of augmented reality. Two questionnaires (pre-test and post-test) are applied to assess student knowledge level on mathematical functions. The pre-test is applied first to both groups and the results obtained show a homogeneous mean. The post-test results show that the students from the experimental group managed to surpass the mean of the control group. It is concluded that the use of augmented reality, implemented as a pedagogical strategy, allowed improving student learning of mathematical functions.
\end{abstract}

Keywords: augmented reality; learning; mathematical functions; pedagogical strategy 


\section{INTRODUCCIÓN}

Las Tecnologías de la Información y las Comunicaciones (TIC) han impactado diversos ámbitos de la vida de los seres humanos y la educación no ha sido la excepción, cada día se exige una verdadera transformación de la práctica educativa, un replanteamiento de las concepciones, la dinamización de los procesos y la renovación de los paradigmas actuales que permita a corto plazo una proyección integral y global. Estos cambios significativos introducidos por las TIC y la globalización vienen desempeñando un papel protagónico y relevante en la educación en la última década. (Solórzanoy García, 2016).Desde esa perspectiva se genera una nueva forma de educar, en donde los actores inmersos deben estar preparados para afrontar los retos impuestos en este nuevo contexto. Dentro de los recursos que brindan las TIC, se encuentran los dispositivos móviles y la realidad aumentada (RA), las cuales permiten crear contenidos educativos capaces de apoyar el proceso de enseñanza-aprendizaje para captar la atención de los estudiantes a los cuales va dirigida la enseñanza (Tovar et al., 2014).

Bajo este panorama, la Realidad Aumentada (RA) como herramienta tecnológica, se ha constituido en un nuevo paradigma y en una aliada que favorece los procesos de formación, toda vez que permite asistir y dinamizar la interacción entre los actores y el mundo real, mediante la conversión de los objetos de la vida cotidiana en recursos interactivos (Buitrago, 2015). De igual manera, contribuye a que los procesos de enseñanza y aprendizaje sean prácticos y tangibles, fomentando la motivación de los estudiantes por la manera como se presentan los contenidos de aprendizaje. La incorporación de esta herramienta en el aprendizaje de la matemática busca que el estudiante desarrolle un pensamiento matemático creativo, crítico e innovador para asegura run aprendizaje significativo y apropiar conocimientos matemáticos a fin de resolver situaciones cotidianas que se presentan en el contexto donde se desenvuelve.Es común encontrar en las instituciones educativas estudiantes que afrontan inconvenientes en el desarrollo del pensamiento matemático y en la interpretación de las funciones, lo que conlleva a un bajo desempeño académico y en algunos casos a la deserción. Este hecho no puede pasar desapercibido para los maestros y las entidades educativas, más aún cuando el estudiante es y debe ser considerado el centro del proceso educativo.

Se podría inferir entonces que al llevar desarrollar un proceso de enseñanza aprendizaje con la utilización de mediaciones TIC, conduce a un aprendizaje dinámico e interactivo que permite la rápida apropiación de situaciones problemáticas. En esta medida, la posibilidad de visualizar gráficamente conceptos teóricos de las funciones matemáticas crea en el estudiante una visión realista de la situación que enfrenta haciendo más real la perspectiva a desarrollar, lo cual favorece el aprendizaje de los estudiantes. En consecuencia, al incorporar la RA en el aprendizaje de las matemáticas, se requiere que los docentes estén preparados y tengan la convicción que la tecnología es una mediación que les permite agilizar y mejorar el proceso educativo; sin embargo, esta debe ser incorporada con una clara intencionalidad pedagógica, entendiéndose que la tecnología por sí sola, no garantiza un adecuado proceso de aprendizaje, por lo que la guía y la orientación del docente, desde esa decisión consciente de aportar al proceso, se torna determinante.

Las tendencias de la educación en la actualidad se unifican al reconocer la importancia de integrar aspectos tales como enseñanzas -aprendizajes significativos, a aprendizaje integral para la vida, metodologías activas, hacerycomprender,usodelasTecnologíasdelalnformaciónylaComunicación(TIC),Tecnologías para el Aprendizaje y el Conocimiento(TAC).Simultáneamente se ha venido desarrollado un dilatado conglomerado de tecnologías específicas en diferentes áreas donde el currículo digital genera una invitación a la integración de innovaciones tecnológicas en la mayoría de asignaturas de enseñanza. (Maquilón,Mirete y Avilés, 2017)

\section{OTROS ANTECEDENTES}

En la actualidad y debido a la relevancia de las TIC en educación por sus efectos positivos, han surgido teorías del aprendizaje e investigaciones que dinamizan este proceso, orientadas a desarrollar en los estudiantes las competencias digitales y específicas en el área de las matemáticas mediadas por la RA, tal como se describe a continuación:

\section{Teorías que soportan la educación mediadas por las TIC}

El conectivismo, teoría de la era digital desarrollada por George Siemens y por Stephen Downes, que transcienden las limitaciones del conductismo, migrando al cognitivismo y luego al constructivismo a fin de explicar el efecto que ejerce la tecnología sobre la manera en que actualmente se vive, se comunica y se aprende (Ovalles, 2014). Está teoría manifiesta un interés en explicar el aprendizaje y sus complejidades en un mundo social digital que evoluciona rápidamente, utilizando el modelo de una red con nodos y conexiones como una técnica de enseñanza-aprendizaje, lo cual implica un cambio de paradigma en la educación basada en la capacidad de conexión entre hechos, ideas, conceptos, redes, actualización del conocimiento, decidir que aprender; donde el eje central del aprendizaje, es el propio individuo que con su deseo de aprender crea su propio ambiente de aprendizaje, conectándose con otros individuos a través de las redes. 


\section{Conectivismo y educación}

El conectivismo es un enfoque teórico que supone una evolución del conocimiento teórico y que de más allá de un mero desarrollo tecnológico, se yergue como una auténtica revolución teórica-practica; es capaz de ofrecer una comprensión adecuada de este tipo de aprendizaje, que surge de la fusión del estudiante con su entorno y la adopción de sus principios, modos de vida y actitudes, en concordancia con el mundo digital interconectado en la actual era digital. (Flórez et al., 2017; Cipresso et al., 2018) Es así como el conectivismo se convierte en una herramienta útil para interpretar y comprender los procesos asociados al aprendizaje, para la adquisición de conocimiento mediado por tecnológica, el trabajo colaborativo mediante diferentes tipos de ambientes de aprendizaje, la mediación y recreación de situaciones de aprendizaje vinculados a las tecnologías a través del e-learning. Es así como el Conectivismo complementa diversas teorías existentes en el campo educativo a modo de adecuación al mundo tecnológico digital a las formas de aprendizaje y adquisición de conocimiento, pero teniendo en cuenta la rápida evolución de la tecnología en este ámbito se requiere la implementación de estrategias dinámicas que permita a estudiantes y académicos tener el debido acceso a estas nuevas modalidades (Álvarez et al., 2017; Flórez et al., 2017)

El Conectivismo, al intentar explicar cómo se adquiere conocimiento y cómo se produce el aprendizaje, compara la mente humana con una red que se adapta al entorno, donde el aprendizaje según esta teoría, se constituye en el proceso de formación mediante redesinterconectadas por distintos nodosy donde el conocimiento residiría en dichas redes. La utilización de los dispositivos móviles genera un alto impacto de aceptación entre los jóvenes estudiantes, que acceden a una variedad de opciones digitales en el momento y en el lugar que lo necesiten, interactuando con los objetos virtuales, con un nuevo estilo de aprendizaje que incorpora la lúdica, tanto en el ámbito formal, como en lo informal (Fombona y González, 2017; Karimi, 2016)

\section{Realidad aumentada}

El estudio de la Realidad Aumentada, a partir de ahora RA, es un desarrollo tecnológico que superpone a una imagen real obtenida a través de una pantalla imágenes, modelos 3D u otro tipo de informaciones generados por ordenador, se inició en la década de los sesenta, en el campo de los gráficos por computadora, cuando se intentó describir la realidad virtual como una ventana a través de la cual un usuario percibe el mundo virtual como si fuera real, esto es, simula una aparente coexistencia entre de virtual con lo real en e tres dimensiones, siendo interactivo en tiempo real y se ha extendido a varias disciplinas. Nace entonces la RA como una nueva tecnología que muestra su eficacia interdisciplinaria en la investigación y proporciona nuevas formas de obtener datos y apoyar el proceso de enseñanza-aprendizaje (Azuma, R., 1997; Prendes, C., 2015; Cipresso et al., 2018)

La Realidad Aumentada se compone de dos elementos: una escena real, en directo (la realidad), y una información adicional asociada a esa escena. La mezcla de estos dos elementos se realiza a través de algún tipo de dispositivo para formar una única imagen que se muestra en una pantalla. Es una tecnología que superpone a una imagen real obtenida a través de una pantalla imágenes, modelos 3D u otro tipo de informaciones generados por ordenador (Prendes, 2015). La RA es aplicable en todas las etapas evolutivas y formativas del ser humano, principalmente en la infancia y en la tercera edad debido a sus condiciones de adaptabilidad en cualquier contexto que se presente. En el ámbito educativo, la RA es una gran aliada para crear una interconexión entre lo virtual y la realidad y ayuda al estudiante a reforzar el aprendizaje y acercarse más a los conocimientos, ya que permite que puedan visualizar de manera completa un problema y lograr una comprensión visual apropiada acerca de este, Por lo que ha llegado a impactar de manera significativa en la resolución de ejercicios, dada la oportunidad de visualizar elementos que apoyen su desarrollo y capacidad de razonamiento (Cabero, Vázquez y López, 2018; Álvarez et al., 2017).

Hoy se aprovecha de manera significativa las bondades que ofrece este tipo de tecnologías para potencializar la comprensión y apropiación de temáticas consideradas abstractas a través de una realidad simulada. Tal como lo plantean Ozdemir et al. (2018), se debe aprovechar las bondades que ofrece la RA como tecnología emergente en la generación de nuevos escenarios de aprendizaje para los estudiantes. Para lograrlo, se requiere contar con una visión general sobre aspectos técnicos, funcionales, los avances, la aplicación y el impacto generado en los entornos educativos, solo así podrá ser incorporada en diferentes niveles educativos de manera coherente y con una verdadera intencionalidad pedagógica. Es así como la RA opera como elemento que favorece las interacciones entre profesor y estudiante dentro de los procesos formales de aprendizaje debido a que permiten acceder a una nueva realidad mediante novedosos recursos que habrán de utilizarse para optimizar estos procedimientos; el profesor asume un rol de guía, que orienta a los estudiantes hacia su propia autonomía, para que estos a su vez asuman la capacidad de crear conocimiento gracias a la doble vía asimilación - exposición que caracteriza el aprendizaje basado en el colectivo de las TIC, tanto del grupo de clase como el colectivo global a través de Internet, permitiendo a los estudiantes mantenerse actualizados en el campo en el cual han formado conexiones. (Ovalles, 2014). 


\section{Realidad aumentada aplicada a la educación}

El desarrollo de la representación del mundo en el transcurso histórico ha ido cambiando de modo coherente con el paradigma de la época, esto es diferentes paradigmas han encarnado las realidades en diversas épocas. En este sentido y en relación a lo tecnológico y digital, el paradigma ha sido cambiado de tal manera que en la actualidad impregna al mundo social, profesional y desde luego al académico.

Bajo el panorama anterior, los usos de la RA en el ámbito educativo y más precisamente en el proceso de enseñanza y aprendizaje, hoy permean diferentes campos del saber y diversos niveles de la educación por adaptarse a las necesidades de los docentes y estudiantes, permitir un mayor acceso a la información mediante esta herramienta tecnológica; por lo cual, se constituye un enriquecimiento ya que genera marcos innovadores de la actividad docente, tales como conformaciones de grupos virtuales, interacciones gratificantes, nuevas escalas de valores, situaciones estas susceptibles de convertirse en fórmulas exitosas del proceso de enseñanza aprendizaje (Fombona y González, 2017). De igual manera, al ser incorporada en un contexto especifico, se debe hacer explícito la intencionalidad pedagógica y los medios digitales, para complementar o aumentar el entorno a través de la información adicional que se pone a disposición (por ejemplo, interfaces visuales, auditivas o táctiles) que tiene relevancia contextual para ese lugar específico (FitzGerald et al., 2013). En el campo de las matemáticas, la visualización a través de la RA, les permite a los estudiantes modular en la realidad los conceptos abstractos que conlleva a una mejor interpretación de los conceptos y su posterior aplicación.

La tecnología de realidad aumentada se ha venido convirtiendo en una de las principales tecnologías para la educación en los diferentes niveles y modalidades educativas. Si bien es cierto que la realidad aumentada es atractiva, es conveniente destacar la inminente necesidad de privilegiar un buen contenido, implementación y evaluaciones, como en todo proceso de integración de tecnología (Baran et al., 2020).Esta nueva realidad del proceso de aprendizaje, enmarcada en la utilización de la realidad aumentada conlleva una experiencia nueva de aprendizaje, frente a otros recursos, lo que puede suponer un aumento en el nivel de comprensión del estudiantado, en la efectividad del proceso de aprendizaje y en la motivación por aprender. Es así como la Realidad Aumentada, modifica lo que se observa para poner en un mismo nivel lo real y lo sintético afianzando el pensamiento creativo de los estudiantes. Por consiguiente, la RA es considerada como un concepto emergente que se encuentra aún en estado inicial de investigación y desarrollo, constituyéndose en uno de los motores más poderosos y con múltiples aplicaciones potenciales en el campo de la educación y más específicamente en el campo de las matemáticas.

Los resultados experimentales de estudios realizados sobre la temática, indican que la integración de RA en las actividades en el aula aumenta la motivación y por ende el logro de aprendizaje de los estudiantes que promueve actitudes positivas sobre temáticas y áreas como la física y las matemáticas, constituyéndose en un foco de interés investigativo. La RA tiene el potencial para apoyar la concreción de un tema, captar el interés de los alumnos y asegurar el aprendizaje. Prueba de ello, son los resultados obtenidos de diferentes investigaciones que ilustran un gran número de implementaciones de la Realidad Aumentada en entornos educativos (Jarmon et al., 2009; McKerlich et al., 2011). En el aprendizaje de las ciencias, por ejemplo, la RA proporciona un gran potencial debido a que permite el uso de varios sentidos, que pueden facilitar el aprendizaje de conceptos científicos abstractos o complejos ( Ozdemir y Col, 2018).

Investigaciones realizadas alrededor de la temática, evidencian que los entornos de aprendizaje con el uso de aplicaciones con RA generan un aumento en la motivación, satisfacción y compromiso de los estudiantes (SaltanyArslan, 2017). De igual manera, muchos de estos estudios destacan el potencial de la Realidad Aumentada (AR) en diferentes dominios educativos y su impacto en los estudiantes con respecto a su mayor motivación, mejor aprendizaje, concentración en el tema (AlptekinyTemmen, 2018).La irrupción de la RA, además de otros instrumentos tecnológicos como elementos transformadores, conlleva a que los docentes asuman dentro de su rol y su formación permanente nuevas metodologías que incorporen en los diferentes niveles de educación estas modalidades (Rodríguez et al., 2019).

\section{RA en los procesos de aprendizaje de las matemáticas.}

La enseñanza de las matemáticas debe ser un proceso contextualizado, creativo en aula y fomentar en el estudiante el razonamiento lógico, la realización de operaciones, la resolución de problemas, el procesamiento de datos y la manera de trasmitir estos, por lo que las didácticas de las matemáticas deberán tener en cuenta elementos esenciales para el desarrollo del pensamiento creativo, la abstracción y la observación, entre otras muchas capacidades. Para ello es fundamental que el docente cuente con herramientas didácticas adecuadas (Hernández et al., 2015). Estas herramientas deben convertirse en uno de los mayores aliados del docente, donde la realidad aumentada es considerada como una de esas herramientas que le permiten al docente tener una técnica novedosa y al estudiante contar con un acompañamiento metodológico y tecnológico. Por 
otra parte a través de experiencias como el uso de dispositivos móviles para el aprendizaje, convierte cualquier escenario en un entorno innovador y creativo modificando el ambiente de enseñanza, tal como ocurre con la RA, que se ha implementado como estrategia de mediación para el aprendizaje de funciones matemáticas en distintas variables generando un efecto positivo en cuanto al logro de aprendizaje en los estudiantes, permitiendo obtener mejores resultados de forma efectiva y significativa (Buitrago, 2015).

Una de las posibilidades que ofrece la RV son los mundos virtuales, que se pueden adaptar para capacitar a los estudiantes en cualquier disciplina, específicamente en matemáticas, donde estos pueden visualizar y manipular problemas mediante un juego virtual de simulación usando sus escritorios como superficies interactivas donde el sistema puede reconocer la interacción multitáctil (múltiples dedos y manos). Adicionalmente, la efectividad educativa de estos juegos virtuales orientados a estudiantes con necesidades educativas especiales demuestra que estos han aumentado sus conocimientos de matemáticas y han participado en las actividades asistiendo con entusiasmo a las lecciones a tiempo. (Le et al., 2014; Papanastasiou et al., 2019)

\section{Funciones matemáticas}

El aprendizaje de la matemática atribuye un papel clave a la interacción social, a la cooperación, al discurso, y a la comunicación, además, de la interacción del sujeto con las situaciones-problemas. El sujeto aprende mediante su interacción con un medio instruccional, apoyado en el uso de recursos simbólicos, materiales y tecnológicos disponibles en el entorno, es allí donde la función se erige como un elemento matemático que muchas veces es capaz de representar realidades cotidianas. (Godino et al., 2003). Cuando se identifican las características individuales y las motivaciones de los estudiantes en la utilización de las plataformas móviles, multidisciplinarmente, indica a los educadores unas prácticas específicas del proceso enseñanza aprendizaje (Karimi, 2016) y específicamente tratándose de las funciones matemáticas, este impulso lúdico en el quehacer de los estudiantes puede vencer obstáculos por difícil que parezca.

En términos generales la problemática asociada al tema se puede resumir como la poca formación docente y falta de recursos financieros para el desarrollo de plataformas y sistemas educativos con el uso de RA, es decir, la información técnica especializada es limitada con respecto a determinados temas sobre RA. De igual manera existe resistencia al cambio de paradigmas y competencia de trabajo colaborativo entre grupos multidisciplinares. Con relación a la variable enseñanza y aprendizaje de las matemáticas es una de las disciplinas que mayor problema presenta, en cuanto a desempeño académico se refiere, en los diferentes niveles de la educación formal. En Colombia por ejemplo, se evidencia una preocupación permanente por parte de los docentes debido al bajo rendimiento del estudiante en matemática en el nivel básico en edad escolar media tal y como lo demostró la prueba PISA en el 2016, donde Colombia obtuvo un promedio de calificación de 370 puntos por debajo del promedio general de los países participantes que fue de 500 puntos mientras que Chile obtuvo un promedio de 450 puntos, lo cual indica que Colombia debe mejorar, y una de las maneras de lograrlo es a través del uso de estrategias didácticas innovadoras por parte de los docentes que logren crear una mayor motivación en los estudiantes por el aprendizaje; en definitiva, la RA como herramienta tecnológica aplicada a la educación, puede aportar métodos y formas innovadoras con el uso de las TIC que mejoren el proceso de enseñanza y aprendizaje visto en la posición del estudiante y del docente.

Sobre lo expuesto, es evidente la importancia y pertinencia que adquiere el uso de didácticas adecuadas y emergentes para la enseñanza de las matemáticas que conlleve a los estudiantes apropiar el conocimiento, toda vez que el aprendizaje de esta área se reviste de gran dificultad para los estudiantes colombianos. Es así como la utilización de la realidad aumentada en educación se ha convertido en una herramienta clave que cuando es implementada como estrategia pedagógica en el aprendizaje de la matemática, se busca que el estudiante se motive y desarrolle un pensamiento matemático creativo, crítico e innovador para asegurar un aprendizaje significativo y apropiar conocimientos matemáticos a fin de resolver situaciones cotidianas que se presentan en el contexto donde se desenvuelvo. Este hecho ha llevado al desarrollo de proyectos de investigación e innovación educativa con el uso RA en esta área, entre los cuales se pueden citar experiencias de autores como: Buitrago (2015) con los proyecto "Un caso para aprender matemáticas"; "Incidencia de la realidad aumentada sobre el estilo cognitivo: caso para el estudio de las matemáticas" realizado en la Universidad de EAFICT -Colombia y el proyecto George (2020), denominado Percepción de estudiantes de bachillerato sobre el uso de Metaverse en experiencias de aprendizaje de realidad aumentada en matemáticas.

Con la investigación se espera que docentes y estudiantes hagan uso de herramientas emergentes durante el proceso de formación que ofrecen amplias posibilidades formativas a fin de lograr el éxito en las aulas y sobre todo aumentar la motivación de los estudiantes por el aprendizaje de las matemáticas con el uso de esta tecnología de forma sencilla, lúdica y formativa a través de la Realidad Aumentada. 


\section{METODOLOGÍA}

En este artículo se presentan y discuten los resultados de investigación llevada a cabo por el grupo Gestión Educativa de la Universidad de la Costa CUC, con el propósito de determinar la incidencia de la Realidad Aumentada en los procesos de aprendizaje de las funciones matemáticas. Se estructuró bajo un enfoque cuantitativo de tipo explicativo, con diseño cuasi experimental con dos grupos experimental y control aplicándose mediciones pre - post test. En el estudio participaron 70 estudiantes de grado $9^{\circ}$ de educación básica de Instituciones educativas de Barranquilla-Colombia, seleccionados de manera no aleatoria e intencional, teniendo como criterio encontrarse matriculados en noveno grado en una de las instituciones educativas seleccionadas para este proyecto. Se utilizó como instrumentos una lista de cotejo haciendo uso del instrumento de revisión de objetos de aprendizaje (LORI) que es una herramienta para validar recursos de aprendizaje multimedia lo que permitió calificar las aplicaciones informáticas de la realidad aumentada mediante criterios como: Calidad del contenido, Alineación de la meta de aprendizaje, Retroalimentación y adaptación, Motivación, Presentación del diseño, Utilidad de navegación, Accesibilidad, Reutilización y Cumplimiento de estándares.

De igual manera se aplicó dos cuestionarios en dos momentos, en el primer momento se aplicó una prueba diagnóstica ( pretest) y en un segundo momento después de haber aplicado la RA en el proceso de formación durante un semestre académico, se aplicó una prueba Postest a fin valorar el nivel de conocimiento matemáticos en el tema de funciones aplicad a los estudiantes del grupo de control y experimental seleccionada para el proyecto; las dos pruebas estuvieron compuestas por 12 preguntas de opción múltiple única respuesta, las cuales fueron agrupadas en 3 dimensiones: Producto cartesiano, Relaciones y Funciones. Los cuestionarios fueron validados por tres (03) expertos y sometido a la prueba de Kuder Richarson con una confiabilidad de 0.84. ya que Según Medina, Ramírez, y Miranda (2019), el índice confiablidad de Kuder-Richardon, es un indicador de la homogeneidad, mide la consistencia interna del test, resulta ser en especial útil porque es utilizable en situaciones de aplicación única de un test o prueba de preguntas de opción múltiple de respuesta única Se utilizó la t de student y la media para apreciar el nivel de conocimiento logrado en la investigación. Los resultados se discuten a la luz de la teoría del conectivismo de George Siemens y Stephen Downes quienes plantean que el aprendizaje es un proceso de conectar nodos o fuentes de información especializado, al igual que los postulados de (Flórez et al., 2017), al considerar que la RA se convierte en una herramienta útil para interpretar y comprender los procesos asociados al aprendizaje y la adquisición de conocimiento en cuanto a la evolución tecnológica, los ambientes de aprendizaje, las mediación y recreación de situaciones de aprendizaje vinculados a las tecnologías a través del e-learning.

Durante un semestre académico se les aplicó a los estudiantes del grupo experimental varios temas tema de funciones matemáticas mediada por RA y accedidos a través de dispositivos móviles o computador de escritorio, donde estos podían observar mediante la APP Aurasma de Realidad aumentada, los objetos e imágenes obtenidas mediante gráficas de estas funciones en 3D. Una vez revisado y apropiado estos recursos se les pedía a los estudiantes resolver de manera individual y grupal actividades de aprendizaje y evaluativas. Los resultado obtenidos durante la aplicación de la RA fueron confrontados con los resultados obtenidos por(Le et al., 2014; Papanastasiou et al., 2019),(SaltanyArslan, 2017), cuando establece quela efectividad educativa de elementos virtuales orientados a estudiantes con necesidades educativas aumenta sus conocimientos de matemáticas, al igual que los entornos de aprendizaje con el uso de aplicaciones con RA, generan un aumento en la motivación, satisfacción y compromiso de los estudiantes.

Durante el desarrollo de la investigación, se diseñaron y desarrollaron bajo el Modelo de Diseño Instruccional ADDIE (Análisis, Diseño, Desarrollo, Implementación y Evaluación) varios recursos educativos digitales multimedia en el tema de funciones en dos variables distribuidos en tres(3) unidades de aprendizaje, estos recursos digitales fueron referenciados en una plataforma (LMS) denominada MANGUS para ser accedidos por los estudiantes tanto del grupo de control ( $\sin$ la RA) como experimental (mediante RA) con el apoyo de los docentes del área; por cada unidad se incorporó dos actividades de aprendizaje y una evaluativa para ser desarrollada con y sin la RA en la temática de funciones a fin de medir el nivel de aprendizaje obtenido por los estudiantes de ambos grupos. Todo ello estuvo soportado en el modelo pedagógico constructivista que permitió tener en cuenta los conocimientos previos de los estudiantes así como el estado inicial de conocimientos de entrada resultante de la prueba diagnóstica aplicada (pretest), los cuales fueron tenidos en cuenta antes de resolver de manera individual y colaborativa las actividades de aprendizaje y evaluativas; y por ultimo este hecho permitió evaluar la incidencia de la RA en el proceso de aprendizaje, acorde con el nivel de desarrollo de las competencias matemáticas logrado durante su formación . Cabe señalar que ambos grupos de control y experimental desarrollan las mismas actividades, solo que el grupo experimental tenía acceso a las gráficas de las funciones en movimiento a través de la RA que permitieron la visualización del modelado de las funciones en más de una dimensión 


\section{RESULTADOS}

Los resultados de la investigación se presentan por cada uno de los objetivos específicos y los instrumentos aplicados a los estudiantes. Éstos se muestran en las tablas 1, figura 1 referidos a la valoración mediante la lista de cotejo de los recursos de RA aplicados en el área de matemáticas y en la tabla 2, figura 2 los resultados del pretest y postest aplicado a los grupos experimental y control.

\section{Identificación de los recursos RA aplicados a la educación en el área de matemáticas}

Una vez revisada en la Web varias herramientas de RA por parte de docentes de las áreas de matemática e informática de las Instituciones Educativas seleccionadas para el proyecto, para su posterior aplicación en el área de matemáticas, fueron seleccionados cinco recursos (Juniao, Layar, Wikitude, Aurasma y Logie T. Rex), por cumplir con ciertos criterios prestablecidos como: contar con el acceso a través de dispositivos móviles inteligentes bajo plataformas Android y iPhone, ser de acceso libre, contar con facilidad de uso y por su aplicabilidad practica al área de las matemáticas. Posterior a la selección de estas 5 herramientas de RA, se procedió a su valoración por parte de docentes y estudiantes mediante el instrumento (LORI) que ya se encuentra validado con nueve criterios o atributos a ser observados, cuyos resultados se plasman en la Tabla 1 , con base en la frecuencia relativa (Fr).

Tabla 1: Valoración de los recursos $R A$ en el área de matemáticas

\begin{tabular}{|c|c|c|c|c|c|c|c|c|c|c|}
\hline \multirow[t]{2}{*}{ Categoría } & \multicolumn{2}{|c|}{$\begin{array}{c}\text { Junaio } \\
\text { www.junaio.es. }\end{array}$} & \multicolumn{2}{|c|}{$\begin{array}{c}\text { Layar } \\
\text { www.layar.com }\end{array}$} & \multicolumn{2}{|c|}{$\begin{array}{c}\text { Wikitude } \\
\text { www.wikitude.com }\end{array}$} & \multicolumn{2}{|c|}{$\begin{array}{c}\text { Aurasma } \\
\text { www.aurasma.com }\end{array}$} & \multicolumn{2}{|c|}{$\begin{array}{c}\text { LogieT.Rex } \\
\text { https://logie.technology/ }\end{array}$} \\
\hline & $F r$ & $\%$ & $F r$ & $\%$ & $F r$ & $\%$ & $F r$ & $\%$ & $F r$ & $\%$ \\
\hline Deficiente & 0 & 0,0 & 0 & 0,0 & 0 & 0,0 & 0 & 0,0 & 2 & 22,20 \\
\hline Regular & 3 & 33,30 & 3 & 33,33 & 3 & 33,33 & 3 & 33,33 & 3 & 33,30 \\
\hline Aceptable & 1 & 11,10 & 2 & 22,20 & 1 & 11,10 & 1 & 11,10 & 2 & 22,20 \\
\hline Bueno & 4 & 44,40 & 3 & 33,33 & 4 & 44,40 & 2 & 22,20 & 1 & 11,10 \\
\hline Excelente & 1 & 11,10 & 1 & 11,10 & 1 & 11,10 & 3 & 33,30 & 1 & 11,10 \\
\hline
\end{tabular}

Al apreciar la Tabla 1 el APP Logie T. Rex es el único en condición deficiente, luego en la condición de regular se encuentran todos los APP con un $33,33 \%$, Layar y Logie T. Rex con un $22,20 \%$ de condición aceptable y los otros con un $11,10 \%$, con un $44,40 \%$ se encuentran en bueno como APP Junaio y Wikitude, y solo Aurasma logro una condición de excelente con $33,33 \%$, por lo cual es el APP utilizado en la presente investigación, lo mismo se puede apreciar en la Tabla 2 y las Fig. 1, que tanto la condición motivación y presentación del diseño del APP para ser aplicado en la realidad aumentada, todos los recursos valorados obtuvieron una calificación de bueno y excelente, sin embargo, se nota que para el recursos RA Aurasma, este resultado es predominante en todos los indicadores analizados, por lo cual, se constituyó en el candidato para ser seleccionado y de esta manera obtener grandes beneficios en el aprendizaje de los estudiantes y lograr que el aprendizaje se produzca de manera lúdica y motivante para los estudiantes.

Tabla 2: Valoración de los recursos $R A$ en el área de matemáticas

\begin{tabular}{|l|c|c|c|c|c|}
\hline \multirow{2}{*}{\multicolumn{1}{c|}{ Criterios }} & \multicolumn{5}{c|}{ APP Realidad Aumentada } \\
\cline { 2 - 7 } & Junaio. & Layar & Wikitude & Aurasma & LogieT.Rex \\
\cline { 2 - 7 } & 1 & 2 & 3 & 4 & 5 \\
\hline Calidad del contenido & 4 & 3 & 4 & 4 & 3 \\
\hline Ajuste del objetivo de aprendizaje & 4 & 3 & 4 & 5 & 1 \\
\hline Retroalimentación y adaptación & 3 & 3 & 3 & 3 & 1 \\
\hline Motivación & 5 & 5 & 5 & 5 & 5 \\
\hline Presentación del diseño APP & 4 & 4 & 4 & 4 & 4 \\
\hline Utilidad de navegación & 4 & 4 & 4 & 5 & 3 \\
\hline Accesibilidad & 2 & 2 & 2 & 2 & 2 \\
\hline Reutilización & 2 & 2 & 2 & 2 & 2 \\
\hline Cumplimiento de estándares & 2 & 2 & 2 & 2 & 2 \\
\hline Total & 30 & 29 & 30 & 32 & 23 \\
\hline
\end{tabular}




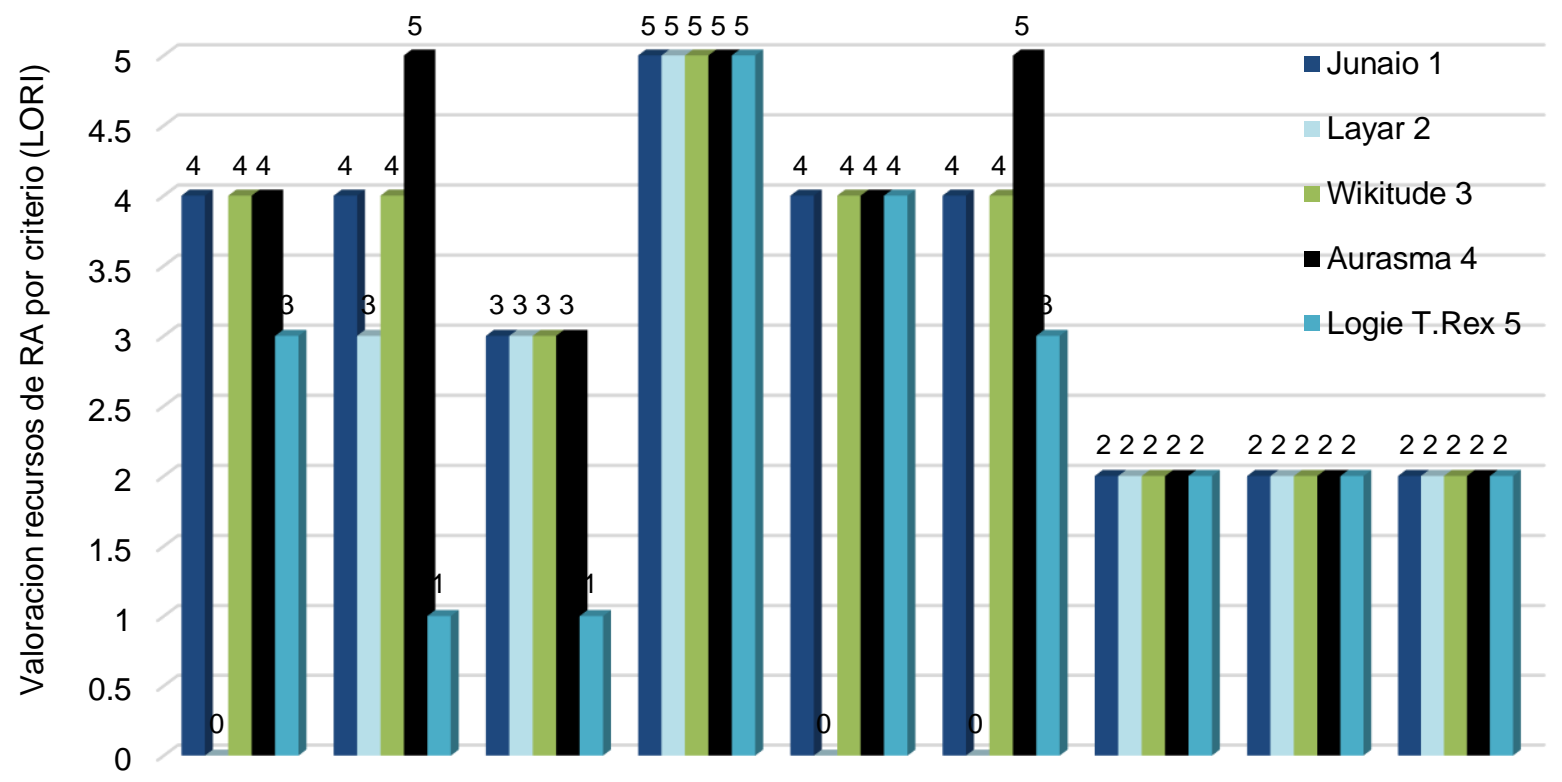

Recursos de Realidad Aumentada

Fig. 1: Valoración de los recursos RA en el área de matemáticas

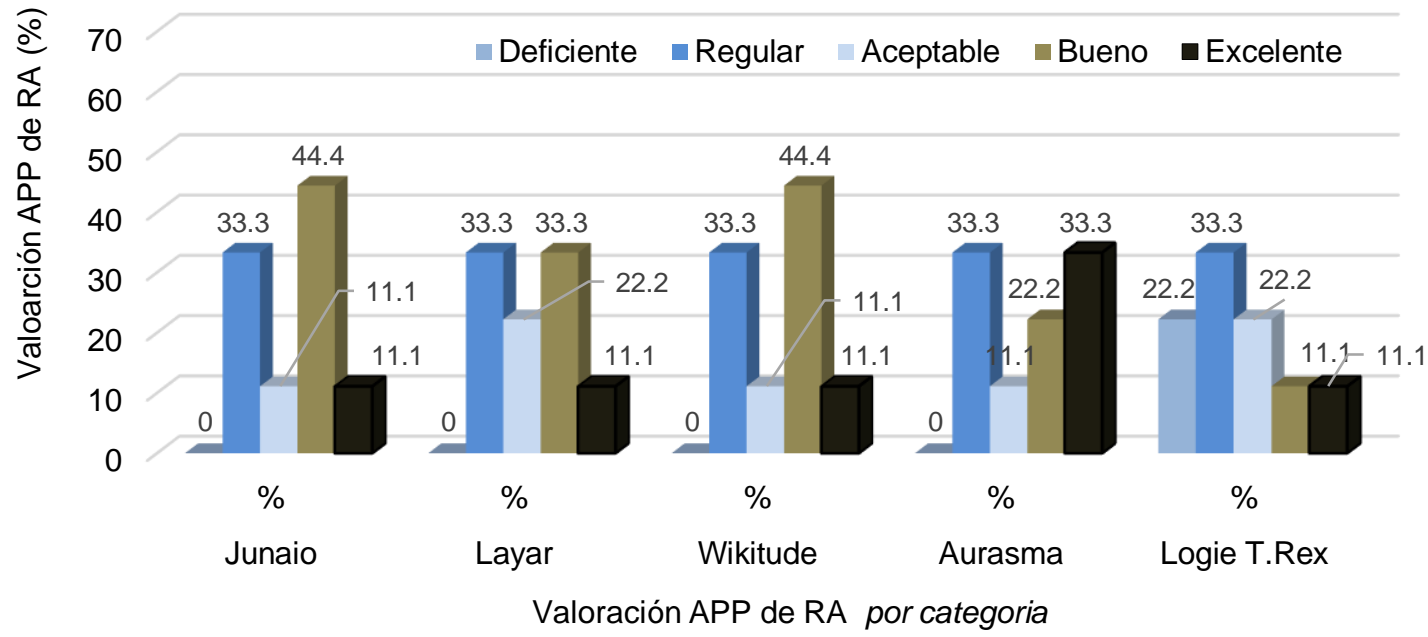

Fig. 2: Resultados de la valoración de los APP

El análisis de los resultados de los test (pretest y postest) aplicados a los dos(2) grupos control y experimental, se realizó a la luz de la definición de los niveles de aprendizaje superficial y profundo planteados por Álvarez Cedillo et al (2019) , quien establece que en el nivel de aprendizaje superficial el estudiante memoriza la información como acontecimientos aislados, sin conexión de experiencias previas o con el entorno general y su objetivo consiste principalmente en asimilar contenidos de manera aleatoria que permita simplemente aprobar la evaluación, lo que explica el rápido olvido de los temas o contenidos estudiados al poco tiempo de haber tenido cualquier evaluación pertinente con la asignatura en diversos contextos de la realidad en el ámbito educativo. A su vez el nivel de aprendizaje profundo corresponde a uno de los más teóricos y aunque puede requerir procesos de búsqueda más profundos para alcanzar la solución, permite mostrar explicaciones mejor elaboradas, analizadas, interpretadas y comprendidas que permitan luego realizar juicios de acuerdo con el entorno.

A continuación, en las Tabla 3 y 4, se describen los resultados de la aplicación del pretest y postest respectivamente aplicado a los grupos experimental y control. La información descrita en la Tabla 3,evidencia el nivel de conocimiento matemático en el tema de funciones en los estudiantes que recibieron clases tradicionales y quienes aprendieron con la realidad aumentada en las Instituciones Educativas seleccionadas para el proyecto, se observa en el grupo de control al realizar el postest, en el indicador superficial que el $57,60 \%$ de las preguntas se respondieron de manera correcta y $42,40 \%$ de forma incorrecta marcando una media de 3,50 que indica un nivel de conocimiento básico; para el indicador profundo se observó que un $54,80 \%$ de las preguntas se respondieron correctamente y $45,20 \%$ de manera incorrecta con media de 3,3 
alcanzando un nivel de conocimiento básico, para una media de 3,4 lo cual indica que el nivel de conocimiento básico reuniendo el superficial y profundo para cuando se dicta la clase tradicional que, planteando una condición no eficiente con las clases tradicionales. Previo al pos-test, se realizó una inducción a los estudiantes para que instalaran en sus equipos móviles, la aplicación Aurasma y luego a través de ejemplos se realizó entrenamiento con el apoyo de los docentes del área de matemáticas e informática, para luego desarrollar ejercicios de funciones matemáticas.

Tabla 3: Pre-test grupo experimental y control (Fa: Frecuencia absoluta; Fr: Frecuencia relativa)

\begin{tabular}{|l|c|c|c|c|c|c|c|c|c|c|c|c|}
\hline \multirow{3}{*}{ Indicador } & \multicolumn{4}{|c|}{ Grupo Experimental } & \multicolumn{4}{c|}{ Grupo Control } & \multirow{2}{*}{ Categoría } \\
\cline { 2 - 16 } & \multicolumn{3}{|c|}{ Correcta } & Incorrecta & Media & Categoría & \multicolumn{2}{|c|}{ Correcta } & Incorrecta & Media & \\
\cline { 2 - 15 } & $\mathrm{Fa}$ & $\mathrm{Fr} \%$ & $\mathrm{Fa}$ & $\mathrm{Fr} \%$ & $\overline{\mathrm{X}}$ & & $\mathrm{Fa}$ & $\mathrm{Fr} \%$ & $\mathrm{Fa}$ & $\mathrm{Fr} \%$ & $\overline{\mathrm{X}}$ & \\
\hline Superficial & 89 & 42,4 & 2,5 & 2,5 & 2,5 & bajo & 97 & 46,2 & 113 & 53,8 & 2,8 & bajo \\
\hline Profundo & 86 & 41,0 & 2,5 & 2,5 & 2,5 & bajo & 80 & 38,1 & 130 & 61,9 & 2,3 & bajo \\
\hline \multicolumn{4}{|c|}{ Media dimensión } & 2,5 & bajo & \multicolumn{4}{c|}{ Media dimensión } & 2.6 & bajo \\
\hline
\end{tabular}

Tabla 4: Post-test grupo experimental y control

\begin{tabular}{|l|l|l|l|l|l|l|l|l|l|l|l|l|}
\hline Prueba & \multicolumn{4}{|c|}{ Grupo Experimental } & \multicolumn{3}{c|}{ Categoría } & \multicolumn{3}{c|}{ GrupoControl } & \multicolumn{3}{c|}{ Categoría } \\
\hline & Fa & Fr\% & Fa & Fr\% & Media & Fa & Fa\% & Fa & Fr\% & Media \\
\hline Superficial & 130 & 61,90 & 80 & 38,10 & 3,71 & básico & 121 & 57,60 & 89 & 42,40 & 3,50 & básico \\
\hline Profundo & 158 & 75,20 & 52 & 24,80 & 8,80 & alto & 115 & 54,80 & 95 & 45,20 & 3,30 & básico \\
\hline Media dimensión & & & & & 6,24 & alto & & & & & 3,40 & básico \\
\hline
\end{tabular}

En el grupo experimental, el indicador superficial de la prueba de pos-test donde se utilizó como herramienta didáctica la realidad aumentada se observa que el 61,90\% de las preguntas los estudiantes las respondió de manera correcta y $34,20 \%$ incorrecta logrando apreciar una media de 3,7 por su parte el indicador profundo muestra que los estudiantes respondieron en un $75,20 \%$ las preguntas de manera correcta y $24,81 \%$ de manera incorrecta y su media fue de 8,80 con un nivel de conocimiento alto. En el pos-test del grupo experimental se muestra una media de 6,24 siendo un nivel de conocimiento logrado alto en su proceso de solucionar problemas de funciones. Es importante también valorar desde la estadística, los resultados de TStuden, representados en la Tabla 5.

Tabla 5: $T$ - Student general de los datos Pos - test

\begin{tabular}{|l|r|r|}
\hline & Grupo de control & Grupo experimental \\
\hline Media & 6,742857143 & 8,228571429 \\
\hline Varianza & 2,608403361 & 4,063865546 \\
\hline Observaciones & 35 & \\
\hline Coeficiente de correlación de Pearson & 0,262492531 & \\
\hline Diferencia hipotética de las medias & 0 & \\
\hline Grados de libertad & 34 & \\
\hline Estadístico $t$ & $-3,945445792$ & \\
\hline $\mathrm{P}(\mathrm{T}<=\mathrm{t})$ una cola & 0,000189263 & \\
\hline Valor crítico de t (una cola) & 1,690924255 & \\
\hline $\mathrm{P}(\mathrm{T}<=\mathrm{t})$ dos colas & 0,000378526 & \\
\hline Valor crítico de t (dos colas) & 2,032244509 & \\
\hline
\end{tabular}

Dado que en la tabla $5, \mathrm{P}(\mathrm{T}<=\mathrm{t})$ dos colas $=0,000378526$ es menor al nivel de probabilidad 0,05 se acepta la hipótesis alternativa que señala que existe una diferencia significativa entre las medias de las calificaciones del grupo de control y grupo experimental por lo cual están heterogéneos, de igual forma como el Estadístico $t=-3,945445792$ es mayor en valor absoluto que el Valor crítico de $t$ (dos colas) $=2,032244509$, queda confirmado que existen diferencias estadísticamente significativa. También se aprecia la diferencia entre las medias del grupo de control $(6,742857143)$ y el grupo experimental $(8,228571429)$ donde el primero recibió clases con clase tradicional y el segundo recibió clase con la estrategia realidad aumenta, y las medias indican el mayor rendimiento del grupo experimental. 
Al contrastar los resultados de esta investigación con los resultados de otros antecedentes realizados por autores como Prendes (2015), donde se evidencia que ambos coinciden en que los grupos que hicieron uso de RA durante el aprendizaje de las matemáticas adquieren una mejora en las calificaciones debido a que se aumenta el grado de motivación; de igual manera con lo planteado por Wilber (2006) quien plantea que en los niveles del conocimiento el sujeto desarrolla un crecimiento psicológico, encontrándose con la aparición de la conciencia de una estructura jerárquica superior, la identificación del yo con la misma, convirtiéndose la nueva en una más compleja, organizada y unificada que la precedente y por ultimo están en concordancia con George, (2020), quien plantea que cuando se utilizan estrategias emergentes mediadas por herramientas de RA favorece el aprendizaje, por tanto, hay afinidad para usar la realidad aumentada en el aula y se lograron mejoras en el índice de aprobación escolar.

\section{CONCLUSIONES}

De acuerdo al análisis de los resultados obtenidos en este estudio, y de su discusión, la comparación con resultados de otros autores quienes establecen la efectividad en el aprendizaje de las matemáticas mediadas por realidad aumentada, se puede extraer las siguiente conclusiones principales: 1) el uso de realidad aumentada a través de APP con acceso móvil, incide de manera positiva en el conocimiento de las funciones matemáticas en los estudiantes, despierta el interés por el aprendizaje, permitiéndoles dedicar mayor tiempo al análisis de la aplicabilidad de las funciones matemáticas en casos problémicos del contexto, es decir, les permite abordar con creatividad cada problema presentado para su solución, 2) El aprendizaje de las matemáticas con la realidad aumentada, conlleva a que los estudiantes avancen del nivel de conocimiento superficial orientado a la comprensión del contenido y al cumplimento de una tarea por parte del estudiante a un nivel de conocimiento profundo donde los estudiantes además de comprender el contenido presentado, busca la relación de nuevas ideas con el conocimiento base, haciendo análisis, comparaciones discerniendo mediante los juicios con la experiencia cotidiana, para posteriormente hacer conclusiones partiendo de la lógica de los argumentos.

\section{REFERENCIAS}

Alptekin, M. y Temmen, K., Design concept and prototype for an augmented reality based virtual preparation laboratory training in electrical engineering, https://doi:10.1109/EDUCACION.2018.836334, Engineering Education Conference (EDUCON), 963-968 (2018)

Álvarez, A., Castillo, M., Pizarro, J. y Espinoza, E., Realidad aumentada como apoyo a la formación de ingenieros industriales, https://doi:10.4067/S0718-50062017000200005, Formación Universitaria, 10(2), 31-42 (2017)

Álvarez, J., Álvarez, T., Sandoval, R. y Aguilar, M., La exploración en el desarrollo del aprendizaje profundo, https://doi.org/10.23913/ride.v9i18.474, RIDE Revista Iberoamericana para la Investigación y el Desarrollo Educativo, 9(18), 833-844 (2019)

Azuma, R. A survey of augmented reality, https://doi:10.1162/pres.1997.6.4.355, Virtual and augmented reality, 6(4), 355-385 (1997)

Baran, B., Yecan, E., Kaptan, B., y Paşayiğit, O., Using augmented reality to teach fifth grade students about electrical circuits, https://doi:10.1007/s10639-019-10001-9, Education and Information Technologies, 25(2), 1371-1385 (2020)

Buitrago, R., The influence of augmented reality on cognitive style: A case for learning mathematics, https://doi.org/10.5294/edu.2015.18.1.2, Educación y Educadores, 18(1), 27-41(2015)

Cipresso, P., Chicchi, I., Alcañiz, M. y Riva, G., The past, present, and future of virtual and augmented reality research: a network and cluster analysis of the literature, https://doi.org/10.3389/fpsyg.2018.02086, Frontiers in psychology, 9, 2086 (2018)

Cabero, J., Vázquez, E., y López, E., Uso de la realidad aumentada como recurso didáctico en la enseñanza universitaria, Formación Universitaria, 11(1), 25-34 (2018)

FitzGerald, E., Ferguson, R., y otros cuatro autores. Augmented reality and mobile learning: the state of the art, https://doi: 10.4018 / ijmbl.2013100103, International Journal of Mobile and Blended Learning, 5(4), 43-58 (2013)

Flórez, S., Pérez, J. y Amaya, Á., Sinergia entre e-Learning y e-Commerce, Tecnología Investigación y Academia, 5(1), 91-106 (2017)

Fombona, J., y González, M., M-learning y realidad aumentada, https://doi.10.3916/C52-2017-06, Revista Científica de Comunicación y Educación, 25(52), 63-72 (2017)

George, C., Percepción de estudiantes de bachillerato sobre el uso de metaverse en experiencias de aprendizaje de realidad aumentada en matemáticas, https://doi.org/10.12795/pixelbit.74367, Revista de Medios y Educación, (58), 143159(2020)

Godino, J., Batanero, C., y Vicenç, F., Fundamentos de la enseñanza y el aprendizaje de las matemáticas para maestros, Universidad de Granada, (2003) 
Hernández, L., García, M., y Mendivil, G., Estrategia de enseñanza y aprendizaje en matemáticas teniendo en cuenta el contexto del alumno y su perfil de egreso, Asesoría entre pares: ¿un método para aprender a aprender a enseñar matemáticas?, Boletín redipe, 4(12), 45-58 (2015)

Jarmon, L., Traphagan, T., Mayrat, M. y Trivedi, A., Virtual world teaching, experiential learning, and assessment: an interdisciplinary communication course in second life, https://doi.10.1016/j.compedu.2009.01.010, Computers \& Education, 53(1), 169-182 (2009)

Karimi, S., Do learners' characteristics matter? an exploration of mobile-learning adoption in self-directed learning, https://doi.10.1016/j.chb.2016.06.014.Computers in human behavior, 63, 769-776 (2016)

Le, Q., Pedro, A., y Park, C. , A social virtual reality-based construction safety education system for experiential learning, https://doi:10.1007/s10846-014-0112-z, Journal of Intelligent \& Robotic Systems, 79(3-4), 487-506 (2014)

Maquilón, J., Mirete, A. y Avilés, M., La realidad aumentada (RA), Recursos y propuestas para la innovación educativa, https://doi.org/10.6018/reifop/20.2.290971, Revista Electrónica Interuniversitaria de Formación del Profesorado, 20(2), 183-203 (2017)

McKerlich, R., Riis, M., Anderson, T. y Easman, B., Student perceptions of teaching presence, social presence and cognitive presence in a virtual world, Online Learning and Teaching, 7(3), 324 (2011)

Medina, J., Ramírez, M., y Miranda, I., Validez y confiabilidad de un test en línea sobre los fenómenos de reflexión y refracción del sonido, http://dx.doi.org/10.32870/Ap.v11n2.1622, Revista Apertura, 11(2), 104-121 (2019)

Ovalles, L., Conectivismo, ¿un nuevo paradigma en la educación actual?, Mundo Fesc, 1(7), $72-79$ (2014)

Ozdemir, M., Sahin, C., Arcagok, S. y Kaan, M., The effect of augmented reality applications in the learning process: A meta-analysis study, https://doi:10.14689/ejer.2018.74.9, Eurasian Journal of Educational Research, 18(74), 165-186 (2018)

Papanastasiou, G., Drigas, A. y otros tres autores, Virtual and augmented reality effects on K-12, higher and tertiary education students' twenty-first century skills, https://doi:10.1007/s10055-018-0363-2, Virtual Reality, 23(4), 425-436 (2019)

Prendes, C., Realidad aumentada y educación: análisis de experiencias prácticas, Revista medios y educación, https://doi.org/10.12795/pixelbit.2015.i46.12, Revista de Medios y Educación, 46, 187-203 (2015)

Rodriguez, C., Sanz, M. y Alonso, S., Technology and higher education: abibliometric analysis, https://doi.org/10.3390/educsci9030169, Education Sciences, 9(3), 169 (2019)

Saltan, F., y Arslan, O., The use of augmented reality in formal education: A scoping review. Eurasia Journal of Mathematics, https://doi.org/10.12973/eurasia.2017.00628a, Science \& Technology Education, 13(2), 503-520 (2017)

Solórzano, F. y García, A., Fundamentos del aprendizaje en red desde el conectivismo y la teoría de la actividad, Revista Cubana de Educación Superior, 35(3), 98-112 (2016)

Tovar, L., Bohórquez, J., y Puello, P., Propuesta metodológica para la construcción de objetos virtuales de aprendizaje basados en realidad aumentada, https://doi:10.4067/S0718-50062014000200003, Formación Universitaria, 7(2), 11-20 (2014)

Wilber, K., La salud mental desde la perspectiva integral, Revista arbitrada, ISSN 0123-417X, Scielo (2006) 
